\title{
Adjustment to Risk Free Rate/ Violation of Put-Call Parity
}

\author{
Saied Simozar ${ }^{1}$ \\ ${ }^{1}$ PhD., Head of Research: Fipmar, Inc., United States \\ Correspondence: Saied Simozar, PhD., Head of Research: Fipmar, Inc., United States.
}

Received: September 16, 2019

Accepted: October 2, $2019 \quad$ Available online: October 17, 2019

doi:10.11114/aef.v6i6.4521

URL: https://doi.org/10.11114/aef.v6i6.4521

\begin{abstract}
The present value of a forward contract for any asset that does not pay a dividend is calculated by discounting its forward price by the risk-free rate. We show that the discount function for assets that have a non-zero correlation with interest rates, has to be adjusted to account for the correlation between the asset and interest rates. Put-Call parity is also violated and needs to be adjusted as well for such assets. It is shown that the risk-free rate is asset dependent. The adjustment to the price is small for short dated forwards, but increases quadratically with time to maturity.
\end{abstract}

Keywords: put-call parity, discount function, risk-free rate, options, forward pricing

\section{Introduction}

The discount function based on risk free rate is the most widely used equation in finance and has been in existence for centuries without any change. Every day trillions of notional dollars trade in currencies, swaptions and financial futures and forwards by applying the discount function to calculate their present values. Unlike stochastic discount function which is used for analysis of risky assets, these transactions are considered to be risk free and the discount function is based on the risk-free rate. While majority are for short dated forwards, there is significant trading on long dated swaptions and some index futures.

One of the applications of the discount function is in the put-call parity equation which can be used to explain, in an intuitive way, how the interaction between an asset price and interest rates can affect the discount function. The put-call parity is an equation with a European long call and a short put with identical strike price and expiration date on one side and a self-financing synthetic forward contract at the strike price on the other side of the equation. When the strike price is equal to the forward price of the security, the forward prices of the call and put are equal. The argument is that we can multiply the forward price of the call or put by the discount function, based on risk free rate, to calculate its present value. This argument as we will see, is not valid for assets that are correlated with interest rates. Nearly all financial assets show some level of positive or negative correlation with interest rates.

Consider an asset whose price is positively correlated with interest rates such as an equity index and let us analyze an option on the asset. Suppose that we build a tree of all possible forward paths of the asset price until the expiration date and we discount each path by its respective interest rate. For paths that result in a call to be exercised, the implied path of interest rates will also point to higher rates due to the positive correlation, implying that the forward premium will have to be discounted by higher rates, resulting in a lower present value for the call than discounting it by the currently prevailing interest rate. The opposite is true for a put option.

The idea of discounting forward paths of an asset price by the then prevailing interest rates is not new and it has been used in the binomial lattice models of interest rates since 1970s. The binomial lattice model proposed by Cox, Ross and Rubinstein (1979) is simple and straightforward to implement and is used widely for pricing path dependent exercise options such as American options. The tree can be constructed by using equal probability for up and down price moves and calculating the prices at the resulting nodes in such a way to match the volatility and arbitrage free requirement. Alternatively, it can be constructed by a constant factor up or down and adjusting the probabilities for each case to match the observed volatility and maintain arbitrage free requirement. See Rubinstein (2000).

Ho and Lee (1986) were among the first to propose an arbitrage free interest rate model based on normal distribution of rates. As such, negative rates are also permitted in the model which is not desirable. Black, Derman and Toy (1990) and the more general model of Heath, Jarrow and Morton (HJM) (1992) use lognormal distribution of rates. HJM model uses non-recombining trees and is significantly more complex and computationally intensive than other models. What is 
common among all these models is that the expected cash flow at every node in the interest rate tree is discounted at the node's prevailing interest rate. This means that the cash flows of an interest rate call option are discounted on average at lower interest rates than those of a put and thus, an at-the-money call option should have a slightly higher value than that of a put.

It appears that there is no reported case of an interest rate call being more valuable than a similar put in the literature. Binomial lattice models converge rather slowly and may not have the accuracy to clearly show that interest rate calls are more valuable than puts, especially for short dated options. There is also extensive calibration that is done at every layer of the tree to ensure arbitrage free requirement. It is possible that some of these calibrations were designed to ensure that put-call parity would not be violated.

There has been a wealth of empirical and quantitative research on stochastic interest rates and their impact on options prices of other assets. Many of these models are based on the work of Amin and Jarrow (1992) who used uncorrelated risky assets along with stochastic interest rates and thereby generalized Black and Scholes (BS) (1973) model that assumes a static discount rate. Rindell (1995) demonstrate that the Amin and Jarrow (1992) model outperforms BS (1973) model by using Swedish option market with expiration dates of up to two years.

Cheng, Nikitopoulos and Schlögl (2015) studied long-dated commodity derivatives within the HJM (1992) framework by incorporating stochastic interest rates that allow a correlation between the commodity futures and interest rates. They found that for long dated options, when the futures price process and the interest rate process are negatively correlated, the call option price is more expensive than the option prices computed when ignoring this correlation. Their findings are consistent with our model's results; however, their analysis is for call options only and there is no mention of the violation of put-call parity in their model or its implication for the discount function.

When the economy slows or recession is forecasted, nominal rates and stock prices fall and corporate bond spreads widen. Likewise, when corporate fundamentals improve or during periods of rapid growth or pricing power by companies, nominal yields and stock prices tend to rise and spreads tend to fall. For these reasons the correlation between rates and stock prices has generally been positive and between rates and spreads has always been negative. Duffee (1998) through regression analysis has shown that there is a negative correlation between interest rates and the level of spreads for all maturities and bond ratings

If interest rates and asset prices were static, the discount function would not need any correction. It is the interaction between rates and asset price changes that needs to be analyzed to calculate the adjustment to the discount function. Thus, we will need to study the stochastic process of forward paths of interest rates just as is done for bond options in the binomial trees, for our analysis.

Among the most popular models for pricing European bond options is a modification of the

BS (1973) model developed by Black (1976) known as Black-76 model. Black-76 uses BS model for equities on interest rates. Because the traded security for an interest rate instrument is the underlying bond, the resulting distribution of bond prices by Black-76 model is not arbitrage free and not useful for long dated options. On the other hand, binomial models suffer from a slow convergence rate that is proportional to $\sqrt{N}$ where $N$ is the number of steps and are of limited use.

While the primary focus of this paper is on the discount function, its application to options markets and the adjustment to put-call parity will likely be one of its biggest uses and it is where its effect can be best quantified. We will first prove that there is an imbalance on the right- hand side of put-call parity for an at-the-money option when it is discounted to the present time. We develop a closed form solution for this imbalance which can be analytically calculated if the volatilities are known.

Next, we work on the left-hand side of the put-call parity equation by using the Black Scholes stochastic rate process, similar to Black-76 model and imposing arbitrage free requirement as a boundary condition to the solution of Black-Scholes differential equation. In the section that follows, we develop an analytical model for discounting the future value of an option that is a function of the future exercise price of the option. This function can be used in a closed-form solution for accurate calculation of European bond options and assets whose returns are correlated with interest rates. This point was quantitatively discussed in Simozar (2015) for bonds and swaptions. However, in this paper we will expand the derivation and provide a more straightforward and general formula for all assets.

It is shown that our analysis of the right-hand and left-hand sides of the put-call parity which are completely independent of each other agree very well. The adjustment to the discount function increases quadratically with the expiration of the option or forward.

\section{Assumptions}

Our first order of business is to explicitly state the assumptions that will be used throughout this paper. The assumptions 
are all considered to be standard assumptions in finance. Some of the assumptions are "strict" assumptions, i.e., if they are violated the derivation and conclusions of the paper will be invalid. Some other assumptions are "loose" assumptions and are used for convenience and if they are violated, the general conclusions of the paper will still be valid but the derivations may have to modified.

1. Arbitrage Free Requirement - One of the ways to state this requirement, which is the cornerstone of all pricing algorithms is that "the expected value of the price of an asset over all possible future paths, is equal to its forward price". Alternatively, the expected value of change in the forward price of an asset is zero given that everything else other than the spot price of the asset is constant. Strict.

2. Geometric Brownian Motion (GBM) - We assume that distributions or processes that are used in this paper follow GBM. If the distributions have fat or skinny tails, the derivations have to be modified, but the general conclusions are valid. This also implies constant volatility (standard deviation) at all forward points in the forward distribution. Loose.

3. Correlation - Correlation between two assets is stable and known. The correlation for two assets $a$ and $b$ with volatilities of $\sigma_{a}$ and $\sigma_{b}$ defined as $\langle\Delta a \Delta b\rangle /\left(a b \sigma_{a} \sigma_{b}\right)=\rho \Delta t$. If correlation is variable but doesn't change its sign the arguments are still valid but the formulations need to be modified. Loose.

4. Flat Yield Curve - Assume that the yield curve or discount function is flat. Loose.

5. Non-Divided Paying Assets - Assume that the assets that are analyzed are non-divided paying. Loose.

6. Efficient and Liquid Markets - Assume that the markets are infinitely liquid, there is no restriction for short selling and no taxation and transaction costs are zero. Semi-Strict.

\section{Objective}

The primary objective of this paper is to show that risk-free-rate cannot be used to calculate the forward price of assets that are correlated with interest rates. Considering that nearly all financial assets and most commodities and currencies are correlated with interest rates, the forward price calculation requires adjustments. The adjustment is small for short duration forwards, but increases quadratically with time to the forward point. If risk-free-rate needs to be adjusted, so will any other equation that relies on the risk free rate, such as put-call parity.

\section{Risk Free European Bond Options}

The price of a risk-free bond with cash flows $c_{i}$, at time $t_{i}$ with a yield $y_{i}$ can be written as

$$
p_{t}=\sum_{i} c_{i} e^{-y_{i} t_{i}}
$$

We assume that forward bond yields $(y)$ in (1) or asset prices $(S)$ follow a geometric Brownian motion (GBM) with time dependent drift, i.e.,

$$
\begin{aligned}
& d \ell n(y)=\mu_{y}(t) d t+v_{y}(t) d B_{y}(t) \\
& d \ell n(S)=\mu_{s}(t) d t+v_{s}(t) d B_{S}(t)
\end{aligned}
$$

Where $\mu_{y}$ is the drift, $v_{y}$ is the volatility and $B_{y}$ is a Brownian motion for an interest rate (yield) process. The same parameters for a non-interest rate process is denoted by subscript $s$. By applying Ito's lemma and solving the resulting Black Scholes PDE or simply noting that (2) represents a lognormal distribution, we find the probability density function $\psi$ as 


$$
\begin{aligned}
& d \psi_{f, t}=A e^{-\frac{\left(\ln (y)-\ell n\left(y_{f}\right)-\mu_{y}\right)^{2}}{2 \sigma_{y}^{2}}} d \ln (y) \\
& \sigma_{y}(t)^{2}=v_{y}(t)^{2} t
\end{aligned}
$$

There are two boundary conditions that need to be met. The first one requiring that the sum of all probabilities in the forward space is equal to one, namely,

$$
\begin{aligned}
& 1=A \int_{y=0}^{\infty} e^{-\frac{\left(\ln (y)-\ln \left(y_{f}\right)-\mu\right)^{2}}{2 \sigma_{y}^{2}}} d \ln (y) \\
& A=\frac{1}{\sqrt{2 \pi} \sigma_{y}}
\end{aligned}
$$

The second boundary condition is the arbitrage free requirement (Assumption 1) which implies that the expected forward price over all probabilities is equal to the forward price; this establishes the drift parameter $\mu_{y}$ for a given time $t$. For non-interest rate assets with price $S$, it can be written as

$$
\begin{aligned}
& S_{f}=\frac{1}{\sqrt{2 \pi} \sigma_{s}} \int_{s=0}^{\infty} S e^{-\frac{\left(\ln (S)-\ell n\left(S_{f}\right)-\mu_{s}\right)^{2}}{2 \sigma_{s}^{2}}} d \ln (S) \\
& \mu_{s}=-\frac{1}{2} \sigma_{s}^{2}
\end{aligned}
$$

Given that the distribution is using the forward prices, the interest rate contribution is already included in the $S_{f}$. For bonds, we assume that the forward yield distribution is GBM (Assumption 2), however, the arbitrage free requirement applies to the price of the bond, i.e.,

$$
\begin{aligned}
& p_{f}\left(\overline{y_{f}}\right)=\int p_{f}(y) \psi(y) d \ln (y) \\
& p_{f}=\frac{1}{\sqrt{2 \pi} \sigma_{y}} \int_{y=0}^{\infty} p(y) e^{-\frac{\left(\ln (y)-\ell n\left(y_{f}\right)-\mu_{y}\right)^{2}}{2 \sigma_{y}^{2}}} d \ln (y) \\
& \mu_{y} \neq-\frac{1}{2} \sigma_{y}^{2}
\end{aligned}
$$

Where $p_{f}$ is the forward price of the bond at the forward yield $y_{f}$. Thus, the drift parameter $\mu_{y}$ has to be calculated numerically to satisfy the arbitrage free requirement (6) at the forward time $t$.

In either case, the probability distribution function of a GBM for an asset $a$ (rate or price) can be written in a Normal Brownian Motion (NBM) notation by a simple transformation as, 


$$
\begin{aligned}
d \psi_{f, t} & =\frac{1}{\sqrt{2 \pi} \sigma} e^{-\frac{\left(\ln (a)-\ln \left(a_{f}\right)-\mu\right)^{2}}{2 \sigma^{2}}} d \ln (a) \\
& =\frac{1}{\sqrt{2 \pi} \sigma} e^{-\frac{(u-\mu)^{2}}{2 \sigma^{2}}} d u \\
u & =\ln \left(a / a_{f}\right)
\end{aligned}
$$

The forward price of a bond call option can then be written as

$$
C\left(t_{x}\right)=\frac{1}{\sqrt{2 \pi} \sigma_{y}} \int_{0}^{y_{x}}\left(p(y)-p\left(y_{x}\right)\right) e^{-\frac{\left(\ln (y)-\ln \left(y_{f}\right)-\mu_{y}\right)^{2}}{2 \sigma_{y}^{2}}} d \ln (y)
$$

\section{Discount Function and Correlation Definition}

Equation (错误!未找到引用源。) is the expected forward price of a call option. In order to calculate its present value, we need to discount it by the risk free rate $r$. We define the discount function as the present value of a unit payoff at some forward time T, i.e.,

$$
d_{T}=\exp (-r T)
$$

We use $y$ to denote the yield of a bond that can potentially have coupon payments and long maturity, while $r$ represents the risk free rate. Fundamentally, they both represent interest rates.

For a constant risk free rate, the discount function at some time $t$ can be written as

$$
d_{t, T}=\exp (r(t-T))
$$

For $\theta=T-t$, the discount function can be written as

$$
d_{\theta}=\exp (-r \theta)
$$

Likewise, the forward price of an asset $S$ in terms of its present price $S_{0}$ can be written as

$$
\begin{aligned}
& S_{f}(T)=S_{0} \exp (r T) \\
& S(t, T)=S_{f} \exp (-r \theta)=d_{\theta} S_{f}(T)
\end{aligned}
$$

Assume that the asset $S_{f}$ and risk free rate $r$ have a correlation $\rho \in[-1,1]$. By definition,

$$
d B_{r}(t) d B_{s}(t)=\rho d t
$$


Now consider put-call parity,

$$
\begin{aligned}
C(t)-P(t) & =\left(S_{f}-K\right) \exp [-r(T-t)] \\
& =\left(S_{f}-K\right) \exp (-r \theta)
\end{aligned}
$$

Where,

$$
C(t) \quad \text { Call price at time } t
$$

$P(t) \quad$ Put price at time $t$

K Strike Price for both the call and the put

We can see by inspection that at time $t=T$ the put-call parity is valid. At all other times, we can multiply both sides of the equation by the discount function as in (10) to arrive at (12).

Given these definitions, we are ready for our theorem.

\section{Theorem: Put-Call Parity for An Asset That Is Correlated with Interest Rates, Is Not Valid}

We construct a portfolio, $Q$ from the right-hand side of the put-call parity, (12) which we write as,

$$
Q=\left(S_{f}-K\right) \exp (-r \theta)
$$

Now suppose that the forward asset price and interest rates change after a short time, $d t$, i.e.,

$$
\begin{aligned}
& \theta=T-t \rightarrow \theta-d t \\
& S_{f} \rightarrow S_{f}(T)+d S_{f} \\
& r(t+d t) \rightarrow r(t)+d r \\
& C(t+d t) \rightarrow C(t)+d C \\
& P(t+d t) \rightarrow P(t)+d P
\end{aligned}
$$

We insert the changes in (13) and do a Taylor series expansion to the second order. Linear components will only have first order expansion, as follows,

$$
\begin{aligned}
& Q(t)+d Q=\left(S_{f}+d S_{f}-K\right) \exp [-(r+d r)(\theta-d t)] \\
& =\left(S_{f}+d S_{f}-K\right) \exp (-r \theta) \times \\
& {\left[1-d r \theta+r d t+d r d t+\frac{1}{2}(-d r \theta+r d t+d r d t)^{2} \ldots\right]} \\
& =\left(S_{f}-K\right) \exp (-r \theta)+d S_{f} \exp (-r \theta)+d S_{f} \exp (-r \theta) \theta d r \\
& +\left(S_{f}-K\right) \exp (-r \theta)\left(-\theta d r+r d t+\frac{1}{2} \theta^{2} d r^{2} \ldots\right)
\end{aligned}
$$

After subtracting (13) from both sides of the above equation, it simplifies to

$$
\begin{aligned}
& d Q=d S_{f} \exp (-r \theta)-\left(S_{f}-K\right) \exp (-r \theta) \theta d r-d S_{f} d r \theta \exp (-r \theta)+ \\
& +\left(S_{f}-K\right) \exp (-r \theta)\left(r d t+\frac{1}{2} \theta^{2} d r^{2} \ldots\right)
\end{aligned}
$$


Assuming $\sigma_{r}$ and $\sigma_{s}$ are the volatilities of interest rates and the asset price respectively and $\rho$ is the correlation coefficient between the forward price of the asset and interest rates, then, by definition,

$$
\begin{aligned}
& d S_{f} d r=r S_{f} \rho \sigma_{s} \sigma_{r} d t \\
& d r^{2}=r^{2} \sigma_{r}^{2} d t
\end{aligned}
$$

Making substitutions (17) and evaluating (16) at $S_{f}=K$ results in

$$
d Q=d S_{f} \exp (-r \theta)-S_{f} r \sigma_{s} \sigma_{r} \theta d t \exp (-r \theta)
$$

The present value of the cumulative adjustment, (18) between time 0 and $T$, can be calculated by multiplying it by $\exp (-r t)$ and integrating it:

$$
\begin{aligned}
& Q(0)=\int_{0}^{T} e^{-r t} d Q=\int_{0}^{T} d S_{f} e^{-r \theta} e^{-r t}-\int_{0}^{T} r S_{f} \theta \rho \sigma_{s} \sigma_{r} e^{-r \theta} e^{-r t} d t \\
& =e^{-r T} \int_{0}^{T} d S-\rho \sigma_{s} \sigma_{r} r S_{f} e^{-r T} \int_{0}^{T}(T-t) d t \\
& =e^{-r T}\left[S_{f}(T, T)-S_{f}(0, T)\right]-\frac{1}{2} \rho \sigma_{s} \sigma_{r} r S_{f} e^{-r T} T^{2}
\end{aligned}
$$

Arbitrage free requirement implies that the forward asset price is constant over time, i.e., $S_{f}(0, T)=S_{f}(T, T)$.

Inserting this into the above equation, results in,

$$
Q(0)=-\frac{1}{2} \rho \sigma_{s} \sigma_{r} r S_{0} T^{2}
$$

If the correlation between an asset and interest rates is positive, (20) is negative definite, implying that a put is more valuable than a call. Inserting this result into (13), we find

$$
\begin{aligned}
& Q(0)=\left(S_{f}-K\right) \exp (-r T)= \\
& \left(S_{f}-S_{0} e^{r T}\right) e^{-r T}=-\frac{1}{2} r S_{0} \rho \sigma_{s} \sigma_{r} T^{2} \\
& S_{f}=S_{0}\left(1-\frac{1}{2} r \rho \sigma_{s} \sigma_{r} T^{2}\right) \exp (r T)
\end{aligned}
$$

Thus, if the correlation between an asset and interest rates is positive, the expected value of the forward price will be lower than the theoretical forward price that can be calculated by simply adjusting the spot price by the compounding factor of the interest rate. Given that historical correlation between rates and stock prices is positive, an at-the-money put is more valuable than a similar call.

This is a direct result of the correlation between rates and other assets. If the correlation coefficient was zero, then (20) would be zero. It is a second order effect which is similar to convexity. The right-hand side of put-call parity, is a negatively convex portfolio. It is short a bond which is convex and long a forward stock which is not as convex. When rates fall which increases the present value of the forward stock price, the stock price falls and mitigates that gain and vice versa. Less variance in the forward stock price implies lower convexity. Thus, the convexity of the short bond results in a negative convexity. 
Even though in the put-call parity the risk free rate is a fixed rate, the whole transaction is subject to market volatility as both the asset price and interest rates move in real life. Given the negative convexity of the portfolio on the right hand of the put call parity and its implied negative expected value, market movements will likely result in margin calls for an at the money forward contract. The cumulative effects of the margin calls before the expiration date of the option will result in a deficit at the expiration of the option.

A simple numerical example can clarify this point significantly. Consider a stock that is priced at 100 and interest rates at $10 \%$, so that the one year forward price of the stock will be 110 . Suppose that the stock price can be either up $10 \%$ or down $10 \%$ in a short period of time, i.e., the forward price will be at 121 or 99 with a probability of 0.5 each. The expected forward price will be $(121+99) / 2=110$ which meets the arbitrage free requirement. Now assume that interest rates can either move up or down by $2 \%$ in a short period of time. The expected forward price will again be $(112+108) / 2=110$ which will meet arbitrage free requirement. However, assume that the stock price and interest rates are correlated in such a way that if stock price increases by $10 \%$, interest rates increase by $2 \%$ and vice versa. The up price will be $1.12 * 110=123.2$ and the down price will be $1.08 * 90=97.2$, so that the expected value will be 110.2 In this scenario, a put option struck at 110 will be slightly in the money and will be worth a little more than a call at 110 . In the formulation, we assumed that the expected value of the forward asset price was constant to meet the arbitrage free requirement, in the numerical example, we kept the spot asset price constant and allowed the forward price to change. In either case, the put option became more valuable due to market volatility.

\section{Correlated Discount Function}

Before we analyze the paths of discount function for an option, we will show a known property of GBM or NBM that recombines into another similar distribution if it is evolved from a time in the future to a forward time. This exercise will help us find the expected value of an asset's price at some time in the future, knowing its initial and final states.

Consider an asset $a$ at time $t_{0}$ that is transformed to a distribution $\psi(u)$ at time $t_{1}$ with standard deviation and drift of $\sigma_{01}$ and $\mu_{01}$ respectively $\left(\sigma_{01}, \mu_{01}\right)$. Next, assume that $\psi(u)$ is further transformed to $\psi(x)$ at time $t_{2}$ by $\left(\sigma_{12}, \mu_{12}\right)$. Our objective at this point is to find the distribution function at time $t_{2}$, knowing the distribution function at $t_{1}$.

We can write the combined distribution function as

$$
d \psi(x) d \psi(u)=\frac{1}{\sqrt{2 \pi} \sigma_{01}} e^{-\frac{\left(u-\mu_{01}\right)^{2}}{2 \sigma_{01}^{2}}} d u \frac{1}{\sqrt{2 \pi} \sigma_{12}} e^{-\frac{\left(x-u-\mu_{12}\right)^{2}}{2 \sigma_{12}^{2}}} d x
$$

In order to calculate the final distribution $\psi(x)$, we must integrate over all possible states of $\psi(u)$, i.e.,

$$
d \psi(x)=\frac{d x}{2 \pi \sigma_{01} \sigma_{12}} \int_{-\infty}^{\infty} e^{-\frac{\left(u-\mu_{01}\right)^{2}}{2 \sigma_{01}^{2}}} e^{-\frac{\left(x-u-\mu_{12}\right)^{2}}{2 \sigma_{12}^{2}}} d u
$$

After some mathematical manipulation we find, 


$$
\begin{aligned}
d \psi(x) & =\frac{d x}{\sqrt{2 \pi\left(\sigma_{01}^{2}+\sigma_{12}^{2}\right)}} e^{-\frac{\left(x-\mu_{01}-\mu_{12}\right)^{2}}{2\left(\sigma_{01}^{2}+\sigma_{12}^{2}\right)}} \\
& =\frac{d x}{\sqrt{2 \pi} \sigma_{02}} e^{-\frac{\left(x-\mu_{02}\right)^{2}}{2 \sigma_{02}^{2}}} \\
\mu_{02} & =\mu_{01}+\mu_{12} \\
\sigma_{02}^{2} & =\sigma_{01}^{2}+\sigma_{12}^{2}
\end{aligned}
$$

This is a known property of GBM or NBM where the drifts are simply added and the square of standard deviations are added as well. Next, we want to find the expected (average) value of $u$ if we know its final state will be at $x$. From (22) we can write

$$
d \psi(x) \int u d \psi(u)=\frac{d x}{2 \pi \sigma_{01} \sigma_{12}} \int e^{-\frac{\left(u-\mu_{01}\right)^{2}}{2 \sigma_{01}^{2}}} e^{-\frac{\left(x-u-\mu_{12}\right)^{2}}{2 \sigma_{12}^{2}}} u d u
$$

Solving (25) analytically, we find

$$
d \psi(x)<u>=\frac{d x}{\sqrt{2 \pi\left(\sigma_{01}^{2}+\sigma_{12}^{2}\right)}} e^{-\frac{\left(x-\mu_{01}-\mu_{12}\right)^{2}}{2\left(\sigma_{01}^{2}+\sigma_{12}^{2}\right)}} \frac{\left(x-\mu_{12}\right) \sigma_{01}^{2}+\mu_{01} \sigma_{12}^{2}}{\sigma_{01}^{2}+\sigma_{12}^{2}}
$$

Substituting for $d \psi(x)$ from (24), we find,

$$
<u>=\frac{\left(x-\mu_{12}\right) \sigma_{01}^{2}+\mu_{01} \sigma_{12}^{2}}{\sigma_{01}^{2}+\sigma_{12}^{2}}
$$

Making the substitutions from (24) and (5) for $\mu$, we find,

$$
\begin{aligned}
& \frac{<u>-\mu_{01}}{\sigma_{01}^{2}}=\frac{x-\mu_{02}}{\sigma_{02}^{2}} \\
& \frac{<u>+\frac{1}{2} \sigma_{01}^{2}}{\sigma_{01}^{2}}=\frac{x+\frac{1}{2} \sigma_{02}^{2}}{\sigma_{02}^{2}} \\
& \frac{<u>}{\sigma_{01}^{2}}=\frac{x}{\sigma_{02}^{2}}
\end{aligned}
$$

Equation (28) is general for most assets except for interest rates, where we used the approximation for the drift $\mu$ using non-interest rate distribution (5). However, for interest rates, it should still be a very good approximation for small changes. Let us simplify (28) using the assumptions that were made at the beginning of the paper. Assuming constant volatility, we can write 


$$
\begin{aligned}
& \sigma_{01}^{2}=v^{2} t_{1} \\
& \sigma_{02}^{2}=v^{2} t_{2}
\end{aligned}
$$

Substituting these values in (28), we find

$$
<u>=x \frac{t_{1}}{t_{2}}
$$

This is a very simple, yet important derivation for calculating the adjustment to the discount function. From the definition (7), we see that $x=\ln \left(a / a_{f}\right)$ so that when the asset price is equal to its forward price, $x=0$. It states that if $x$ represented a point in the forward distribution where an option would be exercised, we would expect that at time $t_{1}$, the forward price would have moved on average by (30).

Now consider a call option on a bond. If we were using binomial lattice models, we would be discounting the call option at each forward node, at the then prevailing discount rate. Likewise, we want to calculate the average yield that the option would have to be discounted by, if it were going to be exercised at point $x$. We first note that if $y_{f}(t)$ is the instantaneous forward rate at time $t$, the yield of the discount function $y_{d}(T)$ can be written as

$$
y_{d}(T)=\frac{1}{T} \int_{0}^{T} y_{f}(t) d t
$$

If the option expiration is at time $T$, we know from (30) that the logarithm of the instantaneous forward yield at time $t$ has shifted by $x t / T$. Thus, the average expected shift for the discount function will be

$$
y_{d}(T)=\frac{1}{T} \int_{0}^{T} x \frac{t}{T} d t=\frac{x}{2}
$$

Compare this with (30); the average shift for the discount function is half that of the forward bond. This is intuitively reasonable, since at any time $t$ as we approach the exercise time $T$, only $(t, T)$ portion of the discount function is subject to volatility, while the forward bond has full volatility.

Equation (32) would be valid if the yield curve was not flat, but the basis point volatility across the yield curve was constant. If the volatility is not constant across the discount function, but is a known function of time, we can calculate the average volatility to use it in (32) (See Appendix).

We define the standard deviation of the discount function as

$$
<v^{2}>T=\sigma_{d}^{2}
$$


Next, we need to formulate the effect of correlation. Writing (17) in differntial form and multiplying both sides by $d S_{f}$ The correlation can be written as

$$
\frac{d S_{f} d r}{S_{f} r}=\rho \sigma_{s} \sigma_{r} d t
$$

Multiplying both sides by $d S_{f}$ we will have

$$
\begin{aligned}
& \frac{d r}{r}=\rho \frac{\sigma_{r}}{\sigma_{s}} \frac{d S_{f}}{S_{f}} \\
& r=r_{0}\left(\frac{S_{f}}{S_{f 0}}\right)^{\rho \frac{\sigma_{r}}{\sigma_{s}}} \\
& \ln \left(\frac{r}{r_{0}}\right)=\rho \frac{\sigma_{r}}{\sigma_{s}} \ln \left(\frac{S_{f}}{S_{f 0}}\right)
\end{aligned}
$$

For an asset that is correlated with interest rates, if the forward price is $S_{f}$, we expect the forward interest rates will be

$$
\begin{gathered}
x=\ln \left(\frac{S_{f}}{S_{f 0}}\right) \\
r=r_{0} e^{\rho x \frac{\sigma_{r}}{\sigma_{s}}}
\end{gathered}
$$

For the discount function, using (32) we have to replace $x$ with $x / 2$. Thus, if $y_{d}$ is the yield of the discount function, it has to modified by,

$$
y_{d}=y_{d 0} e^{\rho x \frac{\sigma_{d}}{2 \sigma_{s}}}
$$

Where $\sigma_{d}$ is defined in (33). This is our general formula for the exercise price dependent discount function for an asset that is correlated with interest rates.

\section{Application of Discount Function}

We define the discount function $d$ as,

$$
d=e^{-y_{d} t}=e^{-y_{d 0} t \exp \left(\sigma_{d} \rho x / 2 \sigma_{s}\right)}
$$

Equation (38) is a prescription for how to discount the cash flow at a given point at the exercise time of an option on a correlated asset. For example, at a point that an asset price has shifted compared to its forward price by $x=2 \sigma$, the discount yield would have a shift of $\sigma_{d} \rho$. For example, if the discount yield is 5\% and the rate volatility is $20 \%$ and $\rho$ 
is 0.6 , then the yield of the discount function will be $5 \% \exp (20 \% \times 5 \% \times 0.6)=5.03 \%$. This difference is small but its effect increases almost exponentially as the time to the expiration of the option increases.

The closed form formula for the present value of a bond call and put options using (错误!未找到引用源。) and discounting it to $t_{0}$ can be written as

$$
\begin{aligned}
& C\left(t_{0}\right)=\frac{1}{\sqrt{2 \pi} \sigma_{y}} \int_{0}^{y_{x}}\left(p(y)-p\left(y_{x}\right)\right) e^{-\frac{\left(\ln (y)-\ell n\left(y_{f}\right)-\mu\right)^{2}}{2 \sigma_{y}^{2}}} e^{-y_{d} t_{0}} d \ln (y) \\
& P\left(t_{0}\right)=\frac{1}{\sqrt{2 \pi} \sigma_{y}} \int_{y_{x}}^{\infty}\left(p\left(y_{x}\right)-p(y)\right) e^{-\frac{\left(\ln (y)-\ell n\left(y_{f}\right)-\mu\right)^{2}}{2 \sigma_{y}^{2}}} e^{-y_{d} t_{0}} d \ln (y)
\end{aligned}
$$

By assuming a correlation of 1.0 for a bond and its discount function and making the following substitutions,

$$
\begin{aligned}
& x=\ln \left(y / y_{f}\right) / \sigma_{y} \\
& x_{x}=\ln \left(y_{x} / y_{f}\right) / \sigma_{y}
\end{aligned}
$$

It can be simplified to

$$
C\left(t_{0}\right)=\frac{1}{\sqrt{2 \pi}} \int_{-\infty}^{x_{x}}\left(p(y)-p\left(y_{x}\right)\right) e^{-\frac{\left(x-\mu / \sigma_{y}\right)^{2}}{2}} e^{-y_{d} t_{0} \exp \left(\sigma_{d} x / 2 \sigma_{y}\right)} d x
$$

Table 1 has the calculated premium of at-the-money (Strike Price=Forward) options on a non-dividend paying asset using the following parameters in the Black-Scholes model:

$\begin{array}{lc}\text { Price } & 100 \\ \text { Volatility } & 22 \% \\ \text { Rate (Ann) } & 5 \% \\ \text { Rate Volatility } & 20 \% \\ \text { Correlation } & 0.5\end{array}$


Table 1. Option prices of an asset with and without correlation adjustment

\begin{tabular}{|c|c|c:c|c}
$\begin{array}{c}\text { Expiration } \\
\text { Years }\end{array}$ & Forward & Corr & Call & Put \\
\hline & & & & \\
\hline 5 & 128.40 & 0 & 19.43 & 19.43 \\
\hdashline 5 & 128.40 & 0.5 & 18.69 & 20.07 \\
\hdashline 3 & 116.18 & 0 & 15.11 & 15.11 \\
\hline 3 & 116.18 & 0.5 & 14.85 & 15.34 \\
\hline 1 & 105.13 & 0 & 8.76 & 8.76 \\
\hdashline 1 & 105.13 & 0.5 & 8.73 & 8.79 \\
\hline
\end{tabular}

We can see that the calculated premiums for calls and puts are identical if the correlation is zero. However, for a positive correlation, the put is more valuable. For a 5-year option the difference in premiums is about $\$ 1.4$ which is very significant while for a one-year option it is only $\$ 0.05$. Lower asset volatility, correlation, interest rate and interest rate volatility all contribute to lowering the gap between call and put valuation.

If we take the call and put values in Table 1 for a 5-year correlated option and insert them in the put-call parity (20), we find that the present value of the asset price is $100-20.07+18.69=98.62$. This is puzzling since we started with an asset price of 100. The reason for this discrepancy is that we are discounting the asset price and the strike price at the same rate. Considering that the forward asset has a lower convexity than the forward financed strike price, it's earning rate will be less than the spot rate of the discount function and so should be its discount rate. To calculate the effective discount rate for the stock we have to adjust the rate to

$$
(1.0+.05)(98.62 / 100)^{1 / 5}-1=4.7086 \%
$$

In fact, if we calculate the forward strike price based on this rate, i.e., $100(1.047086)^{5}=125.867$, we find that the call and put have the same premium of 19.24.

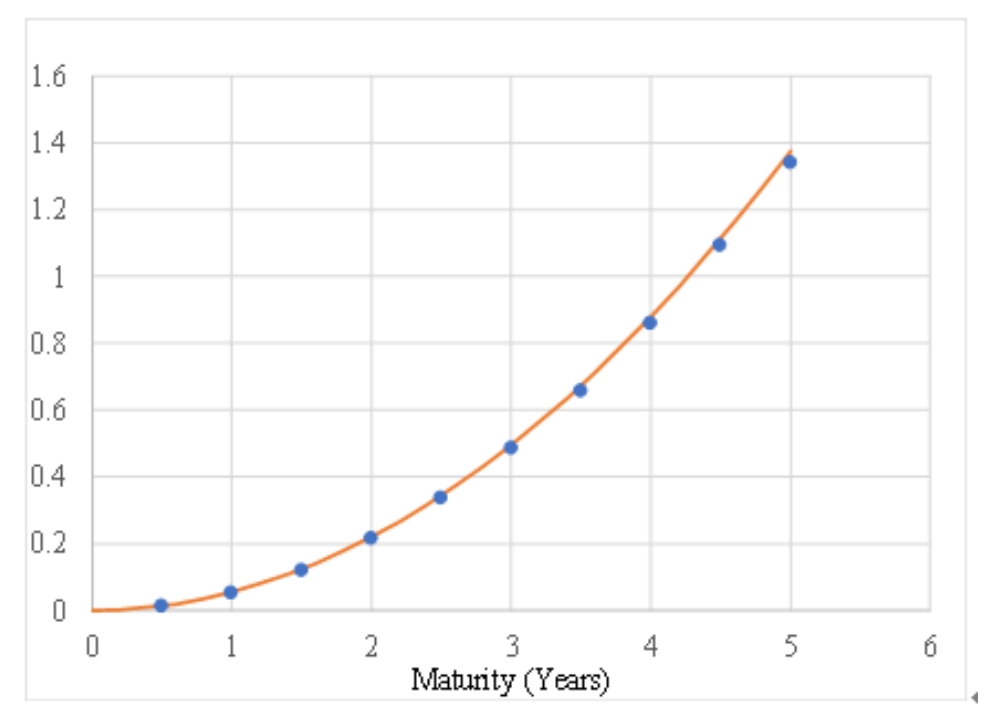

Figure 1. Present value of at-the-money put-call spread \& convexity curve

We calculated the price difference between the present value of the at-the-money put and call and plotted them as a function of the expiration of the option in Figure 1. The solid line is the effect of convexity calculated by (19); the markers are the calculated difference between put and call premium using exercise price dependent discount function (38). The derivation of (19) was completely independent of the calculation of calls and put premiums since we only analyzed the right-hand side of put-call parity. They both agree that the price difference between calls and puts can be 
explained completely by convexity. Figure 1 shows that the effect of correlation on the exercise price dependent discount function is calculated accurately, otherwise the data would not fall on the calculated line.

Given the excellent agreement between (19) and using (38) for option premium calculation, it is logical to assume that (19) can be derived by a Taylor series expansion of (38) and using put and call valuation (39). However, the exponent part of the discount function $\rho x \frac{\sigma_{d}}{2 \sigma_{s}}$ is not necessarily a small number and can't be used in a fast converging Taylor series expansion.

For an option that is out of money, the effect of interest rate on the discount function is larger as a fraction of the option price due to the extreme implied paths of forward interest rates.

Table 2. Option premiums for varying correlation and exercise price

\begin{tabular}{|c|c|c|c|c|c|}
\hline Strike & Corr & Call. & Call Adi. & Put & Put Adi. \\
\hline 155.71 & 0 & 12.26 & & 34.26 & \\
\hline 155.71 & 0.5 & 11.72 & $-4.43 \%$ & 35.19 & $2.69 \%$ \\
\hline 99.55 & 0 & 30.39 & & 8.40 & \\
\hline 99.55 & 0.5 & 29.50 & $-2.93 \%$ & 8.73 & $3.97 \%$ \\
\hline 128.40 & $\ldots$ & 19.43 & & 19.43 & \\
\hline 128.40 & 1 & 17.86 & $-8.08 \%$ & 20.63 & $6.18 \%$ \\
\hline
\end{tabular}

Table 2 has a list of 5-year options that are $(1 \pm 0.22)$ away from the implied forward price of the asset as well as at the money forward. We can see that the out-of-money call has an adjustment of $(11.72 / 12.26-1)=-4.43$ compared to zero correlation case while an in the money call has an adjustment of $(29.50 / 30.39-1)=-2.93 \%$. Likewise, higher correlation results in a higher adjustment but the effect is not linear.

The correlation (convexity) adjusted expected forward price of an asset can, thus be calculated from (19) as

$$
\left\langle S_{f}(T)\right\rangle=S_{0} e^{r T}-S_{0} \int_{0}^{T} r \theta \rho \sigma_{s} \sigma_{r} d t
$$

Alternatively, one can calculate the implied discount rate for a forward asset price as

$$
r=\left(\frac{\left\langle S_{f}(T)\right\rangle}{S_{0}}\right)^{1 / T}
$$

We also calculated the effect of discount function in the swaption market, where we assumed that short rates and long rates are perfectly correlated, but with different volatilities. 
Table 3. Calculated Swaptions prices and comparison with Bloomberg values - June 8, 18

\begin{tabular}{c:c:c|c|c|c|c|c|c|}
\hline $\mathrm{C} / \mathrm{P}$ & $\mathrm{A} / \mathrm{E}$ & \multirow{2}{*}{ Expire } & Vol & Fwd Rate & $\begin{array}{c}\text { Fwd } \\
\text { BBG }\end{array}$ & Prem & $\begin{array}{c}\text { Prem No } \\
\text { Adj }\end{array}$ & $\begin{array}{c}\text { Prem } \\
\text { BBG }\end{array}$ \\
\hline & & & & & & & & \\
\hline $\mathrm{C}$ & $\mathrm{E}$ & $09 / 10 / 18$ & 0.2049 & 3.0298 & 3.0300 & 1.0699 & 1.0697 & 1.0948 \\
\hline $\mathrm{P}$ & $\mathrm{E}$ & $09 / 10 / 18$ & 0.2049 & 3.0298 & 3.0300 & 1.0694 & 1.0697 & 1.0944 \\
\hline $\mathrm{C}$ & $\mathrm{E}$ & $06 / 10 / 19$ & 0.2216 & 3.0596 & 3.0728 & 2.2544 & 2.2489 & 2.3222 \\
\hline $\mathrm{P}$ & $\mathrm{E}$ & $06 / 10 / 19$ & 0.2216 & 3.0596 & 3.0728 & 2.2418 & 2.2489 & 2.3222 \\
\hline $\mathrm{C}$ & $\mathrm{E}$ & $06 / 08 / 23$ & 0.2329 & 3.1450 & 3.1307 & 4.8493 & 4.6693 & 4.8073 \\
\hline $\mathrm{P}$ & $\mathrm{E}$ & $06 / 08 / 23$ & 0.2329 & 3.1450 & 3.1307 & 4.3161 & 4.6694 & 4.8075 \\
\hline
\end{tabular}

Table 3 lists at-the-money (ATM) option premiums calculated for swaptions with forward maturities of 10 years and expiration dates of 3 months, 1 and 5 years on June 8, 2018 and comparing our values with those of Bloomberg's. We used Bloomberg's valuations as a proxy of the broad conventional models. Our forward rates were slightly different from Bloomberg's, which could be due to the timing of the data capture or to methodology. This should have minimal effect on the ATM premiums. We also provide a column for the premiums without adjustment to the discount function for comparison.

For five-year options, the effect of discount function becomes very significant and none of Bloomberg's premiums are reliable. By comparing the premiums with and without adjustments, we can see that the effect of adjustment is not symmetric, largely due to the lognormal distribution of forward rates, where there is no upper bound for rates.

The difference between the call and put premiums when adjusted by the discount function is about $11 \%$.

\section{Volatility Skew}

Black-Scholes (1973) option's pricing model assumes that the volatility is constant for a given option expiry at all exercise prices. Prior to the crash of 1987, equity options had a constant volatility regardless of the strike price. However, after 1987 traded equity options had a higher implied volatility for strike prices that were far from the implied forwards (See Hull, 2003). The higher implied volatility for options that are away from the implied forward price of the asset is known as the volatility smile and it accounts for the fat tails of the probability distribution.

In the fixed income market, there are several methods to model interest rate volatility as a mean reverting process by modifying the rate process (2). Vasicek (1977) used a normal rate process with a mean reverting component to minimize the probability of rates becoming arbitrarily large. Cox, Ingersoll and Ross (1985) use nonlinear stochastic differential equation to combat the negative rates that could result from Vasicek model.

An alternative method to model volatility skew is to modify the probability distribution function (3). This function is a result of a random walk process. Fat tails or volatility smile can be modeled by noting that the step size in the random walk increases as distance from the mean increases. Likewise, in a mean reverting process, the step size decreases as distance increases from the mean. The advantage of modeling volatility inside the distribution function is that the solution is already available and there are no unwanted consequences or complicated or unstable differential equations to solve. The only thing to be done is to renormalize the equation by requiring that the sum of all probabilities is equal to one (4) and the expected forward price over all probabilities is equal to the forward price (5) and (6). With this modification, the Black-Scholes model stays to a large extent intact; only the volatility is replaced by a function that depends on the asset price. While modifying the rate equations might be more appealing from a fundamental point of view, there no a priori reason why this should provide results that are closer to market observations. Additionally, it is significantly easier to reverse engineer the distribution function and deduce the rate process that would lead to the solution.

\section{Conclusion}

The binomial model is the method of choice for many calculations, in particular American options. The binomial model accurately captures the dynamics of the short-term process by discounting every cash flow at its prevailing interest rate. Until now, this was the only way to capture interest rate path dependence on the price of a contingent claim. However, with our model for the analytical method of calculating the exercise price dependent discount function, it might be easier to shift to closed form solutions, where the distribution function is already known.

\section{References}

Amin, K. I., \& Jarrow, R. A. (1992). Pricing options on risky assets in a stochastic interest rate economy. Mathematical 
Finance, 2(4). https://doi.org/10.1111/j.1467-9965.1992.tb00030.x

Black, F. (1976). The pricing of commodity contracts. Journal of Financial Economics, 3. https://doi.org/10.1016/0304-405X(76)90024-6

Black, F., \& Scholes, M. (1973). The Pricing of Options and Corporate Liabilities. The Journal of Political Economy, 81. https://doi.org/10.1086/260062

Black, F., Derman, E., \& Toy W. (1990). A One-Factor Model of Interest Rates and Its Application to Treasury Bond Options. Financial Analysts Journal, 46. https://doi.org/10.2469/faj.v46.n1.33

Cheng, B., Nikitopoulos, C. K., \& Schlögl, E. (2015). Pricing of Long-dated Commodity Derivatives with Stochastic Volatility and Stochastic Interest Rates, Quantitative Finance Research Center, Research Paper 366. https://doi.org/10.2139/ssrn.2712025

Cox, J. C., Ingersoll, J. E., \& Ross, S. A. (1985). A theory of the term structure of interest rates. Econometrica, 53. https://doi.org/10.2307/1911242

Cox, J. C., Ross, S. A., \& Rubinstein, M. (1979), Option Pricing: A simplified Approach. Journal of Financial Economics, 7. https://doi.org/10.1016/0304-405X(79)90015-1

Duffee, G. R. (1998). The relation between Treasury yields and corporate bond yield spreads. Journal of Finance, 53. https://doi.org/10.1111/0022-1082.00089

Heath, D., Jarrow, R. A., \& Morton, A. (1992), Bond Pricing and the Term Structure of Interest Rates: A New Methodology for Contingent Claims Valuation. Econometrica, 60. https://doi.org/10.2307/2951677

Ho, T. S. Y., \& Lee, S. B. (1986), Term Structure Movements and Pricing Interest Rate Contingent Claims. Journal of Finance, 41. https://doi.org/10.1111/j.1540-6261.1986.tb02528.x

Hull, J. C. (2003). Options, Futures and Other Derivatives (5th ed.). Prentice-Hall. p. 335.

Rindell, K. (1995). Pricing of index options when interest rates are stochastic: An Empirical Test. Journal of Banking \& Finance, 19(5). https://doi.org/10.1016/0378-4266(94)00087-J

Rubinstein, M. (2000). On the Relation Between Binomial and Trinomial Option Pricing Models. https://doi.org/10.3905/jod.2000.319149

Simozar, S. (2015). The Advanced Fixed Income and Derivatives Management Guide, Wiley Finance Series, P 221. https://doi.org/10.1002/9781119014157

Vasicek, O. A. (1977). An equilibrium characterization of the term structure. Journal of Financial Economics, 5, 177-188. https://doi.org/10.1016/0304-405X(77)90016-2

\section{Appendix}

To calculate the average volatility of the discount function, which can be different from the volatility of the underlying bond, we first note that the volatility is both a function of time and a function of the maturity of the underlying bond.

Assume that $v\left(t_{v}, t_{f}\right)$ is the instantaneous volatility at time $t_{v}$ of an instantaneous forward yield at time $t_{f}$. Then,

the average volatility of the instantaneous forward yield in the interval $\left(0, t_{f}\right)$ can be calculated from,

$$
<v^{2}>t_{f}=\int_{0}^{t_{f}} v\left(t, t_{f}\right)^{2} d t
$$

To calculate the volatility of the discount function, we need to integrate over all instantaneous forward yields, thus, 


$$
\begin{aligned}
& <v^{2}>\int_{0}^{T} t d t=\int_{0}^{T} d t_{f} \int_{0}^{t_{f}} v\left(t, t_{f}\right)^{2} d t \\
& <v^{2}>=\frac{2}{T^{2}} \int_{0}^{T} d t_{f} \int_{0}^{t_{f}} v\left(t, t_{f}\right)^{2} d t
\end{aligned}
$$

Depending on the model for the term structure of volatility, the instantaneous volatility is generally not known, but the volatility of a forward bond in the interval to the forward point is known. If $w\left(t_{v 1}, t_{v 2}, t_{f}, t_{m}\right)$ is the volatility in the interval $\left(t_{v 1}, t_{v 2}\right)$ of a forward zero coupon bond at time $t_{f}$ with maturity of $t_{m}$ then,

$$
v\left(t_{v}, t_{f}\right)=w\left(t_{v}, t_{v}, t_{f}, t_{f}\right)
$$

The incremental absolute volatility between $t_{v}$ and $t_{v}+d t_{v}$ is calculated as

$$
\begin{aligned}
& w\left(t_{v}, t_{v}+d t_{v}, t_{f}, t_{m}\right)^{2} d t_{v}= \\
& w\left(0, t_{v}+d t_{v}, t_{f}, t_{m}\right)^{2}\left(t_{v}+d t_{v}\right)-w\left(0, t_{v}, t_{f}, t_{m}\right)^{2} t_{v}
\end{aligned}
$$

Thus,

$$
w\left(t_{v}, t_{v}, t_{f}, t_{m}\right)^{2}=\frac{d\left(w\left(0, t_{v}, t_{f}, t_{m}\right)^{2} t_{v}\right)}{d t_{v}}
$$

Equation (5) is general and applies to any forward yield (line segment). For $t_{f}=t_{m}$ and considering equation (3), we substitute from (5) into (2) we find,

$$
\begin{aligned}
& <v^{2}>=\frac{2}{T^{2}} \int_{0}^{T} d t_{f} \int_{0}^{t_{f}} v\left(t, t_{f}\right)^{2} d t \\
& =\frac{2}{T^{2}} \int_{0}^{T} d t_{f} \int_{0}^{t_{f}} d\left(w\left(0, t_{v}, t_{f}, t_{f}\right)^{2} t_{v}\right) \\
& =\frac{2}{T^{2}} \int_{0}^{T} w\left(0, t_{f}, t_{f}, t_{f}\right)^{2} t_{f} d t_{f}
\end{aligned}
$$

This is the formula for calculating the average volatility of the discount function, if we know the volatility of the instantaneous forward yield as a function of time.

\section{Copyrights}

Copyright for this article is retained by the author(s), with first publication rights granted to the journal.

This is an open-access article distributed under the terms and conditions of the Creative Commons Attribution license which permits unrestricted use, distribution, and reproduction in any medium, provided the original work is properly cited. 